\title{
Polysèmes
}

Revue d'études intertextuelles et intermédiales

\section{Le dispositif photolittéraire comme mode de résilience}

Jean-Pierre Montier

\section{(2) OpenEdition}

\section{Journals}

Édition électronique

URL : http://journals.openedition.org/polysemes/3448

DOI : 10.4000/polysemes.3448

ISSN : 2496-4212

Éditeur

SAIT

Référence électronique

Jean-Pierre Montier, «Le dispositif photolittéraire comme mode de résilience », Polysèmes [En ligne], 19 | 2018, mis en ligne le 30 juin 2018, consulté le 21 avril 2019. URL : http://journals.openedition.org/ polysemes/3448; DOI : 10.4000/polysemes.3448

Ce document a été généré automatiquement le 21 avril 2019

Polysèmes 


\title{
Le dispositif photolittéraire comme mode de résilience
}

\author{
Jean-Pierre Montier
}

\section{L'image photographique incline-t-elle en soi à la résilience?}

1 En préalable, il convient de poser un paradoxe initial: l'association même que nous proposons entre deux idées, photographie et résilience, n'est en rien marquée du sceau de l'évidence.

2 Le concept de résilience est entré dans le vocabulaire français courant grâce à Boris Cyrulnik qui, dans un premier temps, en a popularisé l'usage dans le domaine de la psychologie et la psychiatrie (Cyrulnik 1997, 2001, 2004), puis a étendu son emploi jusqu'au récit de ses propres souvenirs d'enfance. Dans Sauve-toi, la vie t'appelle (Cyrulnik 2012), il applique à lui-même la description du mécanisme dit de résilience par lequel il est parvenu à intégrer et dépasser le traumatisme subi un jour de juin 1944 où il est raflé avec d'autres enfants juifs à Bordeaux, mais réussit à échapper à la déportation, au contraire de ses parents qui y trouvèrent la mort. Le succès public de sa production scientifique s'explique par l'incontestable talent de vulgarisateur - au meilleur sens du terme - de Boris Cyrulnik, mais aussi par le fait qu'il est parvenu, au fil de ses ouvrages, à associer trois champs culturels et scientifiques: celui de l'éthologie (l'étude des comportements, y compris celui réputé strictement « instinctif » des animaux), celui de la littérature relative à la Shoah, et celui du récit littéraire relatant une construction de soi, autrement dit ce que l'on dénomme classiquement une autobiographie.

L'on peut donc considérer que la notion de « résilience » (qui signifie étymologiquement à la fois rebondir et résister) est passée en un demi-siècle du domaine des sciences physiques (où elle désignait la capacité d'absorption d'une énergie par un matériau subissant un choc, le caoutchouc par exemple) à celui de la psychologie comportementale - chez Werner et Smith, qui étudièrent à Hawaï dans les années quarante la manière dont des enfants dépassent les troubles dont ils sont atteints grâce à leur environnement social ; 
puis chez John Bowlby qui travailla à partir des années cinquante sur la notion d' attachement et l'importance des liens affectifs dans la construction de $\operatorname{soi}^{1}{ }^{1}$, avant de pénétrer désormais les champs historiques, culturels et littéraires.

Dans le domaine français, c'est en effet la production scientifique de Boris Cyrulnik qui a établi la connexion entre éthologie, histoire collective et littérature, via en particulier ses analyses des survivants des camps de la mort ou de ceux qui, y ayant échappé comme lui, n'en ont pas moins dû apprendre à se reconstruire. Ayant ainsi été à la fois victime, témoin et analyste, Boris Cyrulnik a fait de sa propre histoire un " cas d'école ", à partir duquel la résilience désigne à présent une aptitude éminemment dialectique à absorber et surpasser une situation traumatique, une grave blessure psychique. Tandis que celle-ci aurait "normalement» dû être rédhibitoire et bloquer tout processus ultérieur de développement - et c'est à dessein que nous employons ici une métaphore photographique -, il y a au contraire résilience quand, finalement, au lieu d'avoir pour conséquence une inhibition, un blocage, le choc initial ouvre sur une perspective positive, une reconstruction optimale, un dépassement inespéré, voire exemplaire - et en effet la vie même de Boris Cyrulnik est un exemple de réussite sociale associée à la capacité d'en faire partager la signification universelle.

5 Autrement dit, si la résilience est un processus relevant des capacités des êtres vivants à s'adapter aux conséquences d'un traumatisme, elle suppose un travail (au sens psychiatrique), et donc la mise en place de dispositifs (verbaux pour l'homme) aptes à identifier la nature de la blessure afin d'en amoindrir les effets et de reconstruire un équilibre psychique. Mais pour que ce processus - disons, ce «développement»fonctionne, il ne suffit pas de constater l'existence d'un trauma, bien au contraire. Pour revenir aux camps de la mort, George Rodger avait eu beau photographier Bergen-Belsen en 1945 pour le magazine Life, et David Rousset en décrire par le menu la logique dans L'Univers concentrationnaire, paru en 1946, ni l'un ni l'autre ne rencontrèrent le moindre écho de la part de l'opinion publique, le premier terminant sa carrière comme photographe indépendant en se consacrant exclusivement à l'Afrique, le second ayant été assigné en procès politique par les communistes qui lui reprochèrent d'avoir osé élargir le concept de "camp de concentration" au Goulag soviétique, et participèrent ainsi à l'éclosion du négationnisme.

6 Où est le paradoxe plus haut évoqué ? Il est d'abord dans le constat empirique qu'il ne suffit pas d'apporter des preuves de crimes ou de documenter des exactions pour susciter le moindre travail sur la mémoire. Or, si l'on pose que l'une des valeurs sémiotiques premières de la photographie réside dans sa valeur documentaire ou probatoire, son efficacité en termes de résilience est d'autant plus restreinte qu'elle prête en réalité le flanc aux théories complotistes, quand elle ne les excite pas à se déchaîner ${ }^{2}$. Plus généralement, que l'on considère l'histoire de ses usages ou bien celle de sa critique, la photographie est massivement marquée par tout un ensemble d'idées qui vont $a$ contrario de celle de résilience, celles de nostalgie, de regret, de deuil indépassable: elle encouragerait la contemplation morbide, l'arrêt du temps, le regard toujours rétrospectif, rarement prospectif. Autant la résilience est marquée par l'idée d'aptitude à rebondir et renaître, autant la photographie l'est par les notions d'arrêt, de collapsus (c'est-à-dire, selon l'étymologie latine, la ruine ou la décrépitude), de mélancolie, de déploration, voire de prédation, d'agression (y compris physique). Les photographes eux-mêmes sont davantage concernés par l'idée de chasse que par celle de cure: le «tir intuitif» de Cartier-Bresson est certes nuancé par l'idée de Robert Doisneau que la photographie 
ressemble plutôt à la pêche (attendre le bon moment pour ferrer le poisson ${ }^{3}$ ), mais Chris Marker assume clairement "La photo, c'est la chasse", Diane Arbus parle d'une " occupation diabolique », Frank Horvat assène "Tout photographe est un voyeur », et Michel Tournier, dans Le Roi des aulnes, tranche : «Il est clair que la photographie est une pratique d'envoûtement qui vise à s'assurer la possession de l'être photographié » - pour ne donner que ces quelques exemples parmi un florilège qui serait interminable. L'on épargnera les citations innombrables dans lesquelles l'appareil lui-même est comparé à un phallus agressif. Aussi, sans multiplier les références et citations extraites de la littérature critique, nous bornerons-nous à quelques renvois à un classique, l'ouvrage bien connu de Susan Sontag qui présente une parfaite synthèse de ces vues. Pour elle, "Nous vivons un temps de nostalgie, et les photographies contribuent activement à promouvoir cette conscience nostalgique. La photographie est un art élégiaque, un art de la décadence » (Sontag 25) ; aussi bien, « Toutes les photographies sont des memento mori [qui,] utilisées comme des talismans, sont l'expression d'un état d'esprit à la fois sentimental et implicitement sensible à la magie » (Sontag 26-27) - une magie qu'on suppose plutôt « noire ", ajoutons-nous... Et quand elles sont à même de susciter un désir, loin d'inciter au dépassement de soi et à l'ouverture vers autrui, elles sont les «prototypes de l'objet désirable qui incite à la masturbation» (Sontag 27). Quant aux grandes œuvres photographiques, elles sont pour l'essentiel tournées vers l'irréparable, la ruine et la mort, témoignant des «injures du temps » (Sontag 85) lorsqu'il s'agit de paysages, et ressortissant - quand il s'agit de photographier sur le vif - d'un « caractère abusivement prédateur » (Sontag 139). Dès lors, « [1]a véritable valeur d'une photographie provient du fait qu'elle nous permet d'examiner ou réexaminer à loisir des instants de la durée que le flux du temps emporte aussitôt. Ce pouvoir de congeler le temps [...] a donné naissance à des normes esthétiques plus spécifiquement nouvelles» [par rapport aux normes picturales], car tandis que «les tableaux souffrent du passage du temps [...] l'intérêt qu'on porte aux photographies provient précisément du passage du temps " (Sontag 128-129), et c'est même « ce processus de passage de la durée qui confère de la valeur à n'importe quelle image» (Sontag 159). Pour le dire d'un mot, le régime sémantique convoqué par la technique photographique elle-même, et induit par le type d'image qui en est issu, est estimé fondamentalement régressif.

7 La regrettée Susan Sontag voudra bien pardonner ce cavalier résumé d'une pensée fine, subtile et vive, grâce auquel nous faisons l'économie de renvois multiples à d'autres penseurs tels que Walter Benjamin, Gisèle Freund, Roland Barthes, Rosalind Krauss, JeanMarie Schaeffer, etc., qui tous corroboreraient, sous des angles divers mais convergents, les idées fortes qu'elle expose. Bien entendu, l'on pourra trouver maints exemples de photographies tendant au contraire à la glorification, à susciter l'enthousiasme, à célébrer une victoire, à tendre à la célébration de la beauté du monde, et opposer aux thèses de Susan Sontag le constat qu'on ne photographie pas que des catastrophes ni des morts. Mais quelle conclusion en tirer sinon qu'en effet, dans la logique médiatique de superposition, les images positives peuvent se substituer aux négatives, sans faire autre chose que les masquer, ni contribuer en rien au traitement des traumas? Faut-il vraiment croire que les images de la Libération de Paris le 26 août 1944 annulent par exemple la photo de femmes tondues que Capa a prise à Chartres le 16 août ? S'il est une différence entre ces deux types d'images, elle réside probablement dans leur temps de « développement » respectif : l'image qui glorifie est d'un usage quasi immédiat, tandis que celle qui blesse ou gêne sera initialement oblitérée, puis reviendra éventuellement, avec une latence plus longue, plus obsédante peut-être, car le temps de la victoire n'est 
pas le même que celui des remords ou des crimes cachés - à supposer que l'on souhaite réactiver la trace de ces derniers.

Si à présent nous dirigions notre attention non plus vers les théories de la photographie ni ses emplois médiatisés, mais en direction de ses usages les plus communs, c'est-à-dire les photos prises au sein de la famille, nos conclusions iraient dans le même sens. Certes, la photographie est universellement placée sous le signe du «souvenir » : mais de quoi parle-t-on en invoquant cette vaste et informe catégorie? Dans un texte paru dans le catalogue d'une exposition consacrée aux photographies de famille, André Rouillé souligne que :

Quand les conflits, l'ennui et les drames familiaux transparaissent dans les photographies de famille, c'est en creux, par leurs effets sur les expressions et les attitudes des modèles. [...] Cette énorme production est en fait polarisée par l'enfant, les fêtes rituelles, les activités de loisirs, les lieux familiers, les abords immédiats de la maison, quelques objets quotidiens, et les animaux domestiques. [...] La sexualité et la violence d'une part, les tâches domestiques, le faire en général, d'autre part, lui échappent, tandis que les épisodes douloureux (la maladie, la mort, etc.) n'y occupent qu'une place réduite. (Rouillé n.p.)

Autrement dit, l'activité consistant à photographier est essentiellement conçue en vue de générer la remémoration du meilleur, de recomposer le passé afin de le rendre apte à une célébration très artificielle: il ne s'agit en réalité absolument pas de surmonter des traumatismes grâce à elle, puisqu'elle est comprise par la plupart des personnes privées qui s'y adonnent - et réalisent au moins $98 \%$ des photographies prises dans le monde comme devant par principe gommer tout événement, toute scène ayant un caractère déplaisant, dévalorisant, blessant. Combien fait-on de photographies d'enfants ? Combien de mourants ? Plutôt qu'à les surmonter, les photographies que nous prenons servent au contraire à dénier les véritables traumatismes et les phases anxiogènes de la vie.

\section{Est-il possible de dépasser cette incompatibilité ?}

9 Photographie et traumatisme cependant ont bien en partage l'idée principielle d'un coup, d'une coupure, d'une entaille. Là encore, les références seraient légion : c'est la notion courante du "choc des photos», ou de la photo-choc, qui innerve toute l'histoire du photojournalisme et de la presse de magazines (Lavoie 54-66), mais qui est aussi pensée sous les mêmes auspices chez nombre de théoriciens. Citons seulement Philippe Dubois, qui évoque un " coup de la coupe ", désignant ainsi la double coupure (le double trauma en somme) opérée techniquement et ontologiquement par toute photographie, qu'il définit comme un cut: «Temporellement [...], l'image-acte photographique interrompt, arrête, fixe, immobilise, détache, décolle la durée, en n'en saisissant qu'un seul instant. Spatialement, de la même manière, elle fractionne, prélève, extrait, isole, capte, découpe une portion d'étendue. La photo apparaît ainsi, au sens fort, une tranche, une tranche unique et singulière d'espace-temps, littéralement taillée dans le vif» (Dubois 153, en italique dans le texte). Derechef, les métaphores utilisées par Dubois, sa référence aussi à la figure mythologique de Méduse, déesse aux cheveux de serpents figeant qui la regarde (Dubois 145) orientent en direction de l'effroi, la stupéfaction, absolument pas en direction de la cure, du soulagement, de la guérison. Et de fait, si l'on considère certaines œuvres photolittéraires évoquant la déportation ou la Shoah dans lesquelles des 
photographies sont mentionnées ou reproduites - The Lost, de Daniel et Matt Mendelsohn, ou Un livre du souvenir, de Françoise Milewski ${ }^{4}$-, c'est massivement en tant que preuves qu'elles figurent, et dans le cadre d'enquêtes bien postérieures aux faits, servant d'arguments dirigés explicitement contre les discours négationnistes.

Dans des élaborations littéraires plus complexes, comme $W$ ou le Souvenir d'enfance de Perec, c'est dans un esprit plus ambivalent envers la possibilité de résilience qu'elles interviennent, si l'on suit les analyses de Christelle Reggiani :

Dans $\mathrm{W}$, la photographie rend possible le récit autobiographique précisément parce qu'elle articule la présence à la perte. C'est dire aussi que pour respecter l'écriture de la perte qu'elle permet, pour aller jusqu'au bout de cette essentielle mélancolie, elle doit consentir à sa propre absence : les photographies de $\mathrm{W}$ sont absentes du texte. Le projet perecquien est celui de l'écriture d'une mémoire impossible, que la photographie elle-même ne saurait véritablement refonder. (Reggiani 83)

11 Le paradoxe est ainsi poussé à son comble lorsque l'outil qui devrait servir à se remémorer devient l'instrument sur lequel se fonde désormais une poétique de la disparition, fondée chez Georges Perec sur la conviction qu'il est tout autant impossible de surmonter la blessure originelle que de réaliser, à cause d'elle, une véritable autobiographie. Comment alors imaginer que la photographie, bien qu'elle soit à l'évidence liée au trauma, puisse toutefois s'articuler à son versant positif, à la résilience, à la pacification de la mémoire, ou la reconstruction des mémoires?

Il semble que la raison de ce blocage soit en réalité assez aisée à déterminer. C'est que la photographie, en première approche du moins, ne montre qu'un état, pas les processus, comme le faisait remarquer Bertolt Brecht dans une citation bien connue rapportée par Walter Benjamin: "Une photographie des usines Krupp n'apporte rien sur ces institutions » (Benjamin 27). Mais la fin de la citation est plus rarement prise en compte; Brecht ajoutait que si l'on veut comprendre un phénomène socio-économique tel que la réification: " Il y a donc "quelque chose à construire", quelque chose d'artificiel, de "fabriqué" ». Que convient-il de fabriquer pour qu'une photographie, seule ou en série, soit développable, interprétable, racontable, pour qu'elle ne soit plus un «acte » brut mais devienne un schème discursif, qu'elle permette de remonter une chaîne causale, de réinstaurer un processus génétique, de réintégrer l'événement à une symbolique apaisante, de recomposer un sens ou de restituer une identité brouillée ?

Ce "quelque chose d'artificiel, de fabriqué ", on peut le dénommer dispositif, au sens où Philippe Ortel définit un agencement construit en vue de produire un effet, et articulant des moyens techniques, des échanges pragmatiques (en termes de communication), et des valeurs symboliques (Ortel 35). Cette fabrication, c'est du reste presque chaque jour que Brecht s'y adonnait, dans son Journal de travail (Brecht 1976), découpant des photographies dans des magazines et les analysant longuement ou d'un trait ironique, selon les cas. Mais l'on trouve de lui un exemple plus abouti de dispositif photolittéraire, avec $L ' A B C$ de la guerre, qu'il compose à partir de 1944, et qui rassemble une centaine d'images qu'il dénommait " photogrammes» (des photographies, mais qui étaient dans son esprit analogues à des morceaux d'un film - d'où le terme de " photogramme » - de la guerre que son livre, comme dispositif justement, avait pour vocation de reconstituer). Chaque photogramme est accompagné d'un poème, des quatrains à quatre temps forts rimés deux à deux. L'ouvrage, censuré par les autorités de la RDA au motif de pacifisme en 1950, finit par paraître, expurgé, en 1955. Brecht compose clairement un dispositif, au 
triple sens technique, pragmatique et symbolique, en inventant un livre qui repose sur la tradition allemande des ABC illustrés (de dessins le plus souvent), en reprogrammant leur fonction pédagogique, en détournant les codes du magazine d'actualité dont la plupart de ces photographies montées ou découpées (accompagnées parfois de leurs légendes) sont issues, et en recréant le genre $d u$ "tombeau » poétique avec les pages noires sur lesquelles se détachent en blanc ses poèmes (Brecht 2015). Ici, la fonction thaumaturgique, la visée esthétique tendant à la résilience sont très claires : de par le dispositif conçu, il s'agit littéralement de tourner la page de la guerre mondiale, quitte à choquer en redistribuant les rôles officiels des acteurs, par exemple lorsque Brecht met côte à côte deux photos de tankistes, l'un allemand et l'autre russe, auxquels est consacré un même quatrain qui les réunit au lieu de les opposer. Alors, dans ces conditions, l'image photographique peut en effet incliner vers la résilience.

\section{La construction de la résilience, entre France et Algérie}

14 Toutes choses égales, c'est un dispositif du même type qu'en 2004 conçoit Leïla Sebbar pour Mes Algéries en France $e^{5}$. En termes de technique, ce livre broché d'un format 22x17 comptant 238 pages de papier glacé permet, dans les meilleures conditions possibles, la reproduction des nombreuses illustrations qui le ponctuent (plus d'une centaine). Outre un nombre impressionnant de photographies, personnelles ou d'agences, on trouve des dessins, des peintures, des aquarelles, des cartes postales, des tracts, des publicités. Tous les supports sont convoqués, y compris des fac-similés de pages de manuels de lecture; toutes les époques aussi, du moins des années 1830 à la plus récente actualité (le livre sort six ans après la coupe du monde de football remportée par l'équipe «black, blanc, beur » dont Zidane était capitaine, mais dans un contexte marqué par de multiples émeutes dans les banlieues).

En termes de pragmatique et de communication, c'est la logique du patchwork ou du quilting qui prévaut. Elle est présente dès la couverture : autour du rectangle central sur fond rouge où figurent le titre et le nom de l'auteur, quatorze images sont disposées, des portraits peints, dessinés ou photographiés, de personnages célèbres (Zidane), méconnaissables (Isabelle Eberhardt), fictifs (un zouave dessiné pour une publicité) ou inconnus, parmi lesquels celui de l'auteur enfant (que l'on reconnaît plus loin grâce à une photo de la famille Sebbar réunie au complet). Cette logique du patchwork a une dimension proprement iconologique, c'est celle du collage, de la juxtaposition d'images sans motif apparent, mais aussi de textes, car tous ne sont pas signés de Leïla Sebbar, et divers régimes d'écriture, savants, fictionnels ou journalistiques, sont eux aussi juxtaposés. En examinant de plus près la couverture, l'on s'aperçoit qu'elle légitime des hésitations en termes de classement générique, car la préface de Michèle Perrot, historienne renommée, pourrait lui valoir de figurer parmi les livres d'histoire, tandis que le sous-titre l'oriente vers les guides de voyage - nous l'avions trouvé pour notre part au rayon « tourisme » de notre librairie, une erreur qui n'est pas une incongruité. La table des matières décline sept chapitres, évoquant tour à tour la famille, les femmes algériennes, les arts et lettres, la passion algérienne, les hommes assis, les morts, les jardins de la mère et du père, enfin un bestiaire (abeilles, cigognes, tourterelles...). Les faits et personnages historiques voisinent avec les personnes et les objets de l'intimité, réels ou fictifs. La chronologie est à la fois celle du fil autobiographique (le récit d'une 
enfance en Algérie, que Leïla Sebbar quitte en 1960) et celle du récit historique étalé sur près de 180 ans, les deux étant associés et dépassés grâce au concept de "carnet de voyage ", autorisant le caractère hétéroclite des thèmes ou des figures, les arrêts sur des portraits aussi bien que les réflexions plus abstraites, les retours sur soi et les considérations générales. Rien qui soit cependant contradictoire avec une autobiographie, même au sens où Rousseau l'entendait dans ses Confessions, c'est-à-dire un type d'ouvrage qui ne relèverait pas des mémoires, mais qui serait une sorte d'herbier fait de mots, où l'action consistant à rapporter des souvenirs reviendrait à cueillir des fleurs, collecter des objets qui soient aussi des images porteuses des fragrances du passé. Dans Mes Algéries en France, ce n'est pas la voix de la nature qui est invoquée, ce sont les voix discordantes d'une histoire passée et présente, commune et conflictuelle. L'on y oscille entre l'herbier et le reliquaire, célébration et connaissance, deuil et louange, mais c'est un autre genre qui donne le ton, celui de l'album de photos de famille, avec à l'orée du texte, sur une double page (Sebbar 20-21), les portraits en vis-à-vis de la mère et du père de Leïla Sebbar en noir et blanc, des portraits comme seuls les artisans photographes savaient les faire à l'époque, façon Harcourt. Les images du père algérien et de la mère française posent la question majeure de l'ouvrage: unis et dissemblables, ils sont une origine (celle des vies de leurs enfants) sans être à l'origine du long fil historique qui les a réunis, quoique (ou parce que) dissemblables. Mais faut-il être semblables pour s'unir? $\mathrm{Au}$ contraire, il faut pour Leilla Sebbar apprendre à penser l'un et l'autre, poser l'hétérogène en principe génétique, et savoir à présent pourquoi et comment ré-unir.

Cependant, en termes de symbolique à présent, une figure justifie et légitime ce montage, cet assemblage : celle de la femme qui coud, qui rapièce, ravaude, raccommode, répare. Dans l'iconographie, c'est la photographie d'une publicité pour les machines à coudre 
Singer (Sebbar 65) prise à Paussac, la ville où se rencontrèrent le père et la mère de Leïla Sebbar, qui matérialise la Couseuse.

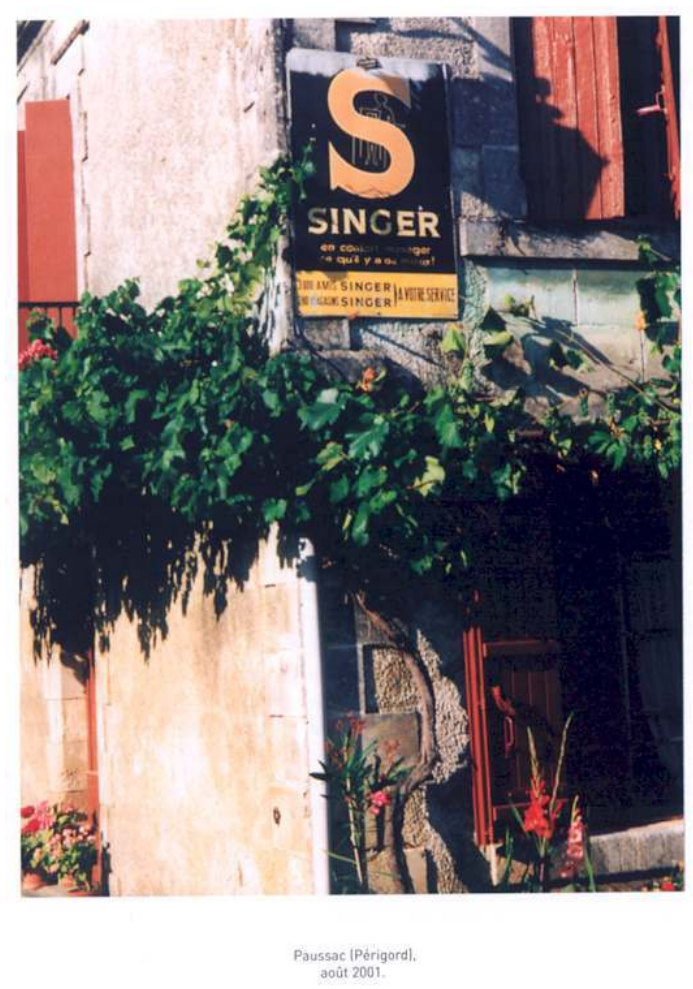

Leilla Sebbar, Mes Algéries en France, Saint-Pourçain, Bleu autour, 2004, p. 65.

(c) Leïla Sebbar

Le S majuscule étant commun à Singer et Sebbar, il devient le dessin ou le monogramme de cette antique figure du monde méditerranéen, associée à la guerre et aux exactions des hommes, mais toujours à l'œuvre, puisque ce sont les blessures, les déchirures qu'il s'agit de repriser. Par-delà les positions féministes de Leïla Sebbar, par-delà aussi les questions relatives à la spécificité hypothétique d'une "écriture féminine ", nous avons là non pas une revendication, ni surtout une posture, mais bel et bien la tranquille affirmation d'une double légitimité, littéraire et esthétique, historique et personnelle, à être sans le moindre hiatus femme et auteur. La figure de la Couseuse, telle que Leïla Sebbar la pose, n'est ni naïve, ni sentimentale, ni ancillaire. Elle ne se soucie guère des questions de genre, la Couseuse étant moins un stéréotype qu'une référence à Pénélope. Leilla Sebbar prouve dans les faits, grâce au dispositif phototextuel tel qu'il est conçu, que c'est comme écrivain et femme qu'elle prend à son compte d'auteur la fonction en effet de résilience en vue de laquelle son Carnet de voyage a été agencé, ourlé, faufilé.

La Couseuse est une figure forte de l'auteur, de même que le tissu est une figure du texte ancrée dans l'histoire culturelle la plus ancienne, mais à la pointe aussi des préoccupations les plus contemporaines quant aux usages et aux mésusages de la mémoire collective et de sa mise en récit ou en œuvre.

19 Mes Algéries en France ne se substitue en aucune manière au manuel d'histoire commune que les historiens des deux pays n'ont jamais été en mesure de rédiger de concert. Sans doute pointe-t-il ce projet jamais abouti comme un manque cruel, en reproduisant les images de plusieurs manuels qui furent conçus du temps de la colonisation, des manuels utilisés à l'école de Bouzaréa par son père, dont Histoire de France et d'Algérie, par Bonnefin 
et Marchand, cours élémentaire, Hachette - la couverture en est reproduite (Sebbar 41) à côté d'un manuel de lecture pour les écoles franco-musulmanes, publié chez Nathan, Bonjour Ali ! Bonjour Fatima !.

Mais après tout, si écrire l'histoire consiste notamment à produire un grand récit, et si nos historiens n'en sont pas capables, n'est-ce pas fondamentalement à la littérature qu'il sied de construire des récits? Certes, nous ne sommes plus au temps de Michelet, inséparablement historien et écrivain; mais ne peut-il revenir à un écrivain de mettre à plat une situation objectivement faite de la juxtaposition de voix disparates et de la concurrence de discours tantôt dogmatiques tantôt hétérodoxes? Cette responsabilité, Leïla Sebbar l'assume pleinement à la fois comme témoin, cobaye même en un sens, assurément comme individu singulier, comme intellectuelle et écrivain, en charge de la transmission d'images et de mots dont le caractère de bien commun, d'une rive l'autre, est tout aussi problématique qu'incontestable.

Il n'y a rien d'irénique dans cette idée de bien commun, de communauté, qui n'est pas à comprendre au sens anglo-saxon d'appartenance à une même chapelle ou secte, mais dans la tradition républicaine française pointant un universel partageable, et nécessitant la recherche, compte tenu de l'hétérogène, d'un dénominateur commun. C'est cette absence d'irénisme qui permet à Leïla Sebbar d'être non pas dans la perspective de ce qu'on appelle " "feel-good" literature ", le recouvrement des mauvais souvenirs par les bons sentiments, mais bien dans la résilience, la formulation et le dépassement du traumatisme. Ainsi utilise-t-elle en somme la pente naturelle de la photographie vers la rétrospection, mais elle en détourne le cours grâce précisément au dispositif cadrant une remise en jeu des discours. C'est le caractère discursif du dispositif photolittéraire qui permet d'inverser le courant herméneutique dans une direction prospective, où les images du passé sont désormais données à interpréter non comme une nostalgie à ressasser, mais comme un patrimoine partageable, quel qu'en soit le poids. Car toutes les photographies ne sont pas mortifères, certaines portent même témoignage d'intenses moments de bonheur, qui même perdus sont à mettre dans la balance de notre héritage. Une photographie, c'est aussi, ontologiquement, ce qui rayonne depuis le passé, comme les sourires des six sœurs Stora déguisées en Berbères sur un balcon à Constantine en 1935 (Sebbar 124-125) ${ }^{7}$. Preuve qu'entre France et Algérie, y compris pour une famille juive, il y eut aussi un "rêve de fraternité ", comme Albert Camus et bien d'autres (le livre rend hommage notamment à Jean Pélégri, Kateb Yacine, Germaine Tillion...) en avaient porté l'intense conviction, un rêve dont le deuil n'est peut-être pas nécessairement définitif, pour peu que l'écriture de l'histoire emprunte d'autres canaux que ceux de l'histoire officielle ${ }^{8}$.

\section{Réparer l'apartheid?}

«Plus tard, elle a travaillé pour une ONG qui l'a chargée d'effectuer des recherches en vue de la rédaction de la nouvelle histoire qui allait être enseignée dans les écoles après la libération » (Vladislavic 94). Cette phrase pourrait être extraite du livre de Leïla Sebbar; or elle l'est du roman d'Ivan Vladislavic, Double négatif, et évoque le passage de l'Afrique du sud à l'ère postapartheid.

Bien entendu ces deux ouvrages n'ont aucun rapport l'un avec l'autre, et a priori rien n'est commun entre les relations France-Algérie et la situation de l'Afrique du Sud. Sauf que la France et l'Algérie, différentes comme nations depuis 1962, possèdent justement en 
partage une mémoire collective fracturée, et que de son côté l'Afrique du Sud s'est constituée dès son origine en une nation traumatisée par la guerre entre les Boers et les troupes anglaises, puis fracturée à nouveau par les lois raciales de l'apartheid.

écrits d'ailleurs

\section{Ivan Vladislavic \\ Double négatif}

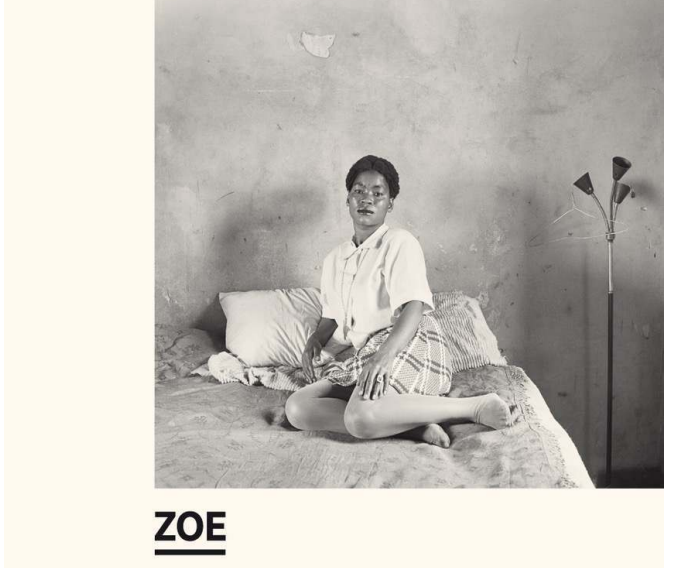

Double négatif, Ivan Vladislavic, édition Zoé, 2013. Photographie de couverture David Goldblatt

Reproduit avec l'aimable autorisation d'édition Zoé

Si le roman d'Ivan Vladislavic ne comporte aucune illustration, sauf une photographie de David Goldblatt en couverture, il fonctionne en réalité en diptyque, couplé avec une exposition et un livre réalisés par le même David Goldblatt, après une série de reportages effectués sur quarante années, essentiellement à Johannesburg et dans la province du Transvaal. Raison pour laquelle le livre et l'exposition de David Goldblatt s'intitulèrent «TJ», les lettres initiales des plaques d'immatriculation des véhicules de cette capitale régionale : «Transvaal Johannesburg ".

Nous avons donc deux œuvres, fonctionnant en diptyque : ce n'est pas en raison du fait que l'écrivain aurait commenté les images du photographe, comme il est classique dans l'édition photolittéraire, mais parce que l'écrivain a fictionnalisé l'entreprise tout entière du photographe, en faisant de ce dernier un personnage à part entière, dénommé Saül Auerbach.

vadisladic va donc raconter en première personne l'histoire d'un jeune homme blanc sud-africain prénommé Neville qui, ne supportant plus la situation d'apartheid, avait choisi de s'exiler en Grande-Bretagne, puis qui revient vivre à Johannesburg après la fin de l'ancien régime. Sans travail, il va passer quelques jours comme assistant auprès de ce photographe célèbre. Il s'agit en un sens d'un roman initiatique : la réalité humaine et sociale sud-africaine étant l'objet de la part de Neville d'un sentiment de dégoût, comment et pourquoi Saül Auerbach - l'alter ego fictif de David Goldblatt - a-t-il pu en faire néanmoins l'objet d'une œuvre ? Comment éviter aussi bien la frivolité consistant à prétendre transmettre des messages que la grossièreté du 
voyeurisme face à la pauvreté ou la souffrance? Saül Auerbach se prête volontiers aux questions de Neville :

"Peut-être que je repère une "image" dans le monde réel quand un de ses éléments reproduit quelque chose que j’ai déjà pensé ou éprouvé.

- Une pièce à conviction.

- À t'entendre, on se croirait en plein crime, mais ce n'est pas le cas. Il n'est pas question de prouver quoi que ce soit non plus. Je ne cherche pas à démontrer un théorème ni à justifier une revendication. Pour parler comme un musicien je cherche des harmoniques. [...] La photographie qui en résulte - à moins que ce ne soit l'acte même de photographie ? - rétablit l'ordre.

- C'est donc thérapeutique? [nous soulignons]

- Non, je n'irais pas jusque là. » (Vladislavic 48)

Clairement, il ne s'agit ni de dénoncer, ni même de s'engager, encore moins de lisser la réalité en la recouvrant de bons sentiments, dans la logique d'une "feel-good" photographie. La fonction de l'art n'est pas directement de guérir («C'est donc thérapeutique? »), comme le confirme plus loin un autre personnage (Vladislavic 91). La solution vient dans le cours de la conversation entre les personnages, avec l'idée singulière que l'Afrique du Sud relevait non du reportage mais de la science-fiction : «C'est une machine à remonter le temps. C'est l'idée que le passé se faisait du futur $-\mathrm{Ou}$ l'inverse, a dit Auerbach » (Vladislavic 49).

Là est le cœur du problème, et l'esquisse de la réponse : si indubitablement associée au réel soit-elle, la photographie produit du vraisemblable, et non du vrai. Sa fonction est analogue à celle de la fiction, qui selon Paul Ricœur est de reconfigurer notre expérience du temps. Alors, le présent factuel, le passé rémanent, le futur pressenti viennent à s'entrechoquer, se compénétrer, s'annuler. Tout se passe comme s'il y avait une forme d'homologie entre ce dont est faite la photographie (qu'on définira comme un « nœud de temps ", voir infra), et la forme que la vie sociale a prise dans ce pays (une société elle aussi faite de « nœuds » mémoriels). Ainsi la « photogénie » de l'Afrique du Sud résulte-telle de l'imbrication de ces nœuds: photographier ce pays, c'est le donner à saisir et comprendre comme une sorte de nœud gordien temporel et sociétal, entre un passé sans racines véritables et un avenir bouché du fait de la permanence de projets idéologiques inspirés d'idées révolues. Il en résulte un pays de « science-fiction », dont le sens ne serait pas dirigé vers son avenir, une machine (infernale) désorientée.

En un sens, le diptyque - autre forme de dispositif - de Vladislavic et Goldblatt croise récit fictif et photographie constative à la manière dont Alfred Stieglitz, réalisant des images de nuages à visée clairement métaphysique, parlait d'équivalents ${ }^{10}:$ une photo n'est ni un constat, ni un procès-verbal, ni une preuve, elle n'accable ni n'héroïse personne; c'est à peine si elle raconte quelque histoire que ce soit, à peine si elle est tout à fait fiable, et pourtant elle est comme un micro-roman en attente de développement. Ne relevant ni du réel, ni du récit, elle joue dans l'interface de l'un et l'autre, équivalent de réel, équivalent de récit. La photographie est un opérateur d'échanges entre des plans ou des registres $a$ priori peu conciliables, un facteur de "transactions " ${ }^{11}$ entre des modèles de représentation ou des schèmes d'expériences. 


\section{Résilience et développement}

30 Il convient probablement de se doter de définitions de la photographie différentes de celles héritées de la sémiologie, dès lors qu'elle intervient à l'intérieur d'un dispositif photolittéraire, c'est-à-dire pour sa capacité en l'occurrence à entrer dans la boucle d'un travail - assurant une forte dimension narrative et de verbalisation - ayant un objectif de résilience. Or, dans un tel dispositif, les photographies tendent selon nous à fonctionner non pas selon des tiroirs temporels - motif pour lequel, étant toujours passées, on a peu à en dire, sinon en effet «ça a été »-, mais plutôt en fonction des catégories aspectuelles (duratif, ponctuel, etc.). Dès lors, ce n'est plus au seul vecteur temporel, linéaire et téléologique, que l'on a affaire, mais bien à de la durée, au sens bergsonien, autrement dit à du subjectif et du qualitatif. En déniant la successivité, en ne suturant pas les coupes qu'elle pratique dans le temps, la photographie noue dans l'espace de l'image les qualités subjectives propres au sentiment de la durée : "La photographie, écrivait Edmond Couchot, est un nœud de Temps qu'on aime à dénouer " (Couchot 106). C'est cette aptitude-là qu'exploitent les œuvres photolittéraires orientées vers la résilience: les nœuds de temps, ou plus exactement les différentiels aspectuels - accompli ou non, semelfactif, ponctuel ou duratif -, sont éminemment utilisables comme équivalents des nœuds dramatiques dont la littérature de son côté se nourrit.

31 C'est la raison pour laquelle, dès l'entame de cet article, nous avancions la notion de "développement », dont ces deux exemples (le livre de Leïla Sebbar et le diptyque de Vladislavic et Goldblatt - mais nous pourrions en réalité les multiplier dans l'édition récente $^{12}-$ ) laissent à penser que ce terme commun à la rhétorique argumentative (un développement dans un discours) et à l'opération de révélation photographique (en français du moins) permet de combiner les deux phénomènes qui paraissent propres à ce genre littéraire singulier, dédié au trauma et sa résilience. L'on ne se trouve pas face à de la littérature illustrée, ni à des photographies commentées, mais devant des opérations plus complexes, relevant d'une combinaison entre le constat factuel, la reconstitution de l'imaginaire collectif, l'énoncé de thèses et d'interprétations historiques, la confrontation des mémoires, la remise en circulation des valeurs partagées et la validation de leur opérativité. Si des œuvres parviennent à conjuguer et dialectiser les opérations sémantiques et sémiotiques à la fois littéraires et photographiques, pourtant si différentes voire conflictuelles, c'est qu'en réalité les unes et les autres, grâce justement à un dispositif réglant leurs interactions, sont mises en situation d'échange, de transaction, entre deux formes de développements: les uns rhétoriques (la discursivité, la narrativité), les autres que l'on pourrait tout simplement qualifier avec Jean-Christophe Bailly de "photoniques » : c'est au fond le même souffle photonique qui nous donne à voir aujourd'hui la célèbre meule de foin de W.F. Talbot et l'ombre d'un homme fixée sur un mur lors de l'explosion d'Hiroshima. Développement ou bien « souffle» (blow up) : «[... ] il y a dans la nature même de l'interruption photographique quelque chose de quasi catastrophique : la césure qui est en toute image photographique contient le programme de la disparition de ce qu'elle suspend et qu'elle sauve » (Bailly 144). Toute photographie, comme le rappelle ici Bailly, est une quasi catastrophe : là est son homologie native avec l'événement traumatique. Ce qu'elle a surpris a toujours disparu, mais elle le sauve en le donnant à développer comme un souffle parvenu depuis le passé, un passé strictement physique, mais qu'il tient toutefois à nous de savoir capter, pour le conjuguer dans notre 
présent avec le sentiment fin de la durée subjective, qui est notre façon de nous sentir inscrits dans ce flux ${ }^{13}$.

En généralisant, nous pourrions dire que les dispositifs photolittéraires mis en place dans le cas des œuvres visant à la résilience ont pour spécificité d'avoir pour objectif de traiter conjointement plusieurs types de temps, ou plutôt en effet de durées. Il ne s'agit pas seulement de réconcilier la mémoire collective avec l'écriture de l'histoire, ni les mémoires individuelles avec l'histoire officielle. Ce n'est pas le temps psychologique et le temps chronologique des historiens qu'il convient de renouer dans la trame d'une fiction commune plus ou moins lénifiante ou optimiste. Car si toute photo est contestable comme preuve de quoi que ce soit, elle est en revanche incontestable plus secrètement, en ce qu'elle appartient au même flux de temps physique que celui auquel, sans l'éprouver à chaque instant, nous sommes cependant tous soumis. Ce temps photonique n'est autre que celui de notre commune incompréhension devant ce qu'il en est des choses, du réel, et singulièrement des événements traumatisants. Réfléchissant ainsi à propos du "Concept de 11 Septembre »- qui a donné lieu à de nombreux ouvrages mettant en place des dispositifs photolittéraires, dont en 2005 le livre de Jonathan Safran Foer, Extremely Loud and Incredibly Close - Jacques Derrida écrit : «L'événement, c'est ce qui arrive et en arrivant arrive à me surprendre, à surprendre et à suspendre ma compréhension. L'événement c'est d'abord que je ne comprenne pas » (Derrida 133). Autrement dit, face à un événement à caractère traumatique, ce sont au moins la mémoire psychologique personnelle et la mémoire historique commune qui sont affectées, et dont les fils sont à ravauder. Mieux : une sorte de troisième mémoire, qui ne relève ni des récits collectifs ni des inconscients personnels, est convoquée par le trauma lui-même :

Il nous faut penser autrement la temporisation d'un traumatisme. [...] L'épreuve de l'événement a pour corrélat tragique non pas ce qui se passe présentement ou ce qui s'est passé, au passé, mais le signe avant-coureur de ce qui menace de se passer. C'est l'avenir qui détermine l'inappropriabilité de l'événement, ce n'est ni le présent ni le passé. Je précise. Il s'agit d'un trauma, et donc d'un événement dont la temporalité ne procède ni du maintenant présent ni du présent passé, mais d'un im-présentable à venir. Une arme blesse et laisse à jamais ouverte une cicatrice inconsciente ; mais cette arme est terrifiante parce qu'elle vient de l'avenir, d'un avenir si radicalement à venir qu'il résiste même à la grammaire du futur antérieur. (Derrida 144)

Si l'événement traumatisant, comme l'analyse si finement Jacques Derrida, est bel et bien de l'ordre de l'à venir davantage qu'un problème de mise à jour du passé revisité par le présent, alors l'on comprend mieux que la complexité et la justification même d'un dispositif photolittéraire ne soit de l'ordre ni de la preuve ou du document, ni du témoignage ou de l'archive : ce dont il s'agit fondamentalement, c'est de faire en sorte que les diverses modalités de notre inscription dans le temps (sous les auspices du récit historique collectif, du récit de soi personnel) soient finalement (en perspective) comme reconnectées avec la temporalité mate, brute, physique, photonique en effet - c'est-à-dire indifférente au bien comme au mal - dans laquelle nous sommes emportés, transportés.

Par-delà le paradoxe premier que nous avions énoncé, là est l'atout irremplaçable de la photographie, de par sa capacité à opérer des transactions entre l'insupportable et le compréhensible, son aptitude à s'en tenir aux faits tout en ouvrant sur des développements fictionnels non pas rassurants mais du moins résilients, et résilients non 
parce qu'ils nous rassurent sur l'avenir, mais parce qu'ils nous réinscrivent dans la nécessité de faire face, en toute circonstance passée ou à venir, à l'irreprésentable.

\section{BIBLIOGRAPHIE}

Bailly, Jean-Christophe. L'Instant et son ombre. Paris : Éditions du Seuil, « Fiction et Cie », 2008.

Benjamin, Walter. «Petite histoire de la photographie ». Trad. A. Gunthert. Études photographiques 1 (nov. 1996) :

https://journals.openedition.org/etudesphotographiques/99

(dernière consultation le 18 mai 2018).

Brecht, Bertolt. Journal de travail. Trad. Philippe Ivernel. Paris : L'Arche, 1976.

Brecht, Bertolt. L'ABC de la guerre. Réédition Philippe Ivernel. Paris : L'Arche, 2015.

Couchot, Edmond. «Prise de vue, prise de temps ». Les Cahiers de la Photographie $8: 106$.

Cyrulnik, Boris. L'Ensorcellement du monde. Paris : Odile Jacob, 1997.

Cyrulnik, Boris. Les Vilains Petits Canards. Paris : Odile Jacob, 2001.

Cyrulnik, Boris. Parler d'amour au bord du gouffre. Paris : Odile Jacob, 2004 [The Whispering of Ghosts: Trauma and Resilience. Trad. Susanne Farfield. London: Penguin, 2005].

Cyrulnik, Boris. Sauve-toi, la vie t'appelle. Paris : Odile Jacob, 2012.

Derrida, Jacques. Le « concept » de 11 Septembre : dialogues à New York (octobre-décembre 2001) avec Giovanna Borradori. Paris : Galilée, 2005.

Dubois, Philippe. L'Acte photographique. Bruxelles : Labor, 1983.

Lavoie, Vincent. Photojournalismes. Paris : Hazan, 2010.

Ortel, Philippe. «Vers une poétique des dispositifs ». Penser la représentation II. Paris :

L'Harmattan, 2005, 33-57.

Reggiani, Christelle. « Perec : une poétique de la photographie ». Littérature 129 (2003) : 77-106.

Rouillé, André. « Clichés de la vie privée. Parcours à la lisière de l'intime », catalogue de l'exposition Photos de famille. Paris, La Grande Halle-La Villette, 1990, sans pagination.

Sebbar, Leïla. Mes Algéries en France, Carnet de voyages. Saint-Pourçain : Bleu Autour, 2004.

Sontag, Susan. La Photographie. Trad. Gérard-Henri et Guy Durand. Paris : Éditions du Seuil, « Essais », 1979 [On Photography, 1973].

Vladislavic, Ivan. Double négatif. Trad. Nida et Christian Surber. Genève : Zoé, 2013 [Double Negative. Milano : Contrasto, 2010]. 


\section{NOTES}

1. Emmy E. Werner \& Tuth S. Smith, Vulnerable but Invincible, New York: McGraw-Hill Book Company, 1982. John Bowlby, Attachement et perte, 3 vol., Paris : PUF, 1978. [Attachment and Loss, New York: Basic Book, 1969]. Voir aussi les travaux de Serban Ionescu incluant les domaines des grandes catastrophes naturelles, des génocides et des dictatures, dans l'ouvrage collectif qu'il a dirigé, Traité de résilience assistée, Paris: PUF, 2011. Ajoutons que le concept s'étend jusqu'au domaine socio-économique chez Oscar Chapital Colchado, La Attribución Causal y las Emociones Negativas, München: Grin Verlag, 2013. Mais il s'applique également à l'informatique ou à la gestion des ressources humaines, pour décrire la capacité d'un système, quel qu'il soit, à résister en cas de « panne ».

2. Le sociologue Pierre-André Taguieff a remarquablement analysé ce phénomène, en particulier dans La Foire aux illuminés, Paris : La Table ronde, 2005. Nous renvoyons également à l'article de Christian Delage consacré à l'exposition Mémoire des Camps, en 2001, dans lequel l'auteur insiste sur la présence d'un « médiateur culturel » au sein du dispositif global de l'exposition elle-même, pour encadrer sa réception. Voir la revue Vingtième Siècle 72.1 (2001) : 143-145.

3. Robert Doisneau, À l'imparfait de l'objectif, Paris : Actes sud, « Babel», 2001, 102.

4. The Lost, Daniel et Matt Mendelsohn, New York: Harper \& Collins, 2006. Un livre du souvenir, à la recherche d'une famille juive décimée en Pologne, Françoise Milewski, Paris : La Découverte, 2009.

5. Signalons que par la suite l'auteur a fait paraître chez le même éditeur, Bleu Autour, un Journal de mes Algéries en France, 2005, éclairant la genèse de l'ouvrage.

6. Il s'agit d'un courant éditorial relativement récent, ou plus exactement récemment identifié comme tel, dans lequel on trouve des livres tels que Le Vieux qui ne voulait pas fêter son anniversaire, de Jonas Jonasson, Muchachas de Katherine Pancol, et des auteurs tels que Marc Lévy ou encore Anna Gavalda.

7. L'histoire de la famille Stora est rapportée dans un texte signé de Marthe Stora, dont les propos ont été recueillis par Leïla Sebbar en 1991, 120-127.

8. Je renvoie à l'ouvrage de Guy Dugas, Algérie, un rêve de fraternité, Paris : Omnibus, 1999. L'on sait que c'est la position intransigeante de Jean-Paul Sartre qui avait marginalisé celle défendue par Albert Camus, qui, depuis les années 2000, voit son œuvre réhabilitée par de nombreuses publications; il est même revendiqué comme "écrivain algérien» par des universitaires algériens (voir notamment le colloque de Guelma, octobre 2013).

9. David Goldblatt, TJ, Johannesburg photographies, 1948-2010, Milano : Contrasto, 2010. L'une des expositions eut lieu à la Fondation Henri Cartier-Bresson, Paris, janvier-avril 2011.

10. Il s'agit du titre générique d'un ensemble d'œuvres considérées comme le testament esthétique d'Alfred Stieglitz, sur le sens desquelles on pourra aller voir Rosalind Krauss, "Stieglitz Equivalents", October 11 (Winter 1979): 129-140, MIT Press, URL: http:// www.jstor.org/stable/778239, dernière consultation 18 mai 2018.

11. Sur ce concept, voir Jean-Pierre Montier, Transactions photolittéraires, Rennes: PUR, 2014, 11-59.

12. Voir la thèse de Angeliki Tseti, «La littérature à l'âge de la reproduction photographique, le cas des récits décrivant la guerre et la douleur ", sous la direction de François Brunet et Théodora Tsimpouki, université Paris-Diderot, 2015.

13. Sur le rôle de la photographie dans la réflexion esthétique et l'œuvre de Jean-Christophe Bailly, voir Jean-Pierre Montier, "L'usage de la photo », revue Europe, "Jean-Christophe Bailly » (juin-août 2016) : 164-174. 


\section{RÉSUMÉS}

Si l'image photographique est fréquemment liée à des scènes ou des événements traumatisants ou blessants, personnels ou collectifs, et alors que l'acte photographique lui-même a souvent été associé à l'idée de prédation, il convient de se demander comment et dans quelles conditions cette image, au-delà de sa valeur de preuve ou de document, peut assurer la dimension curative et pacificatrice de la résilience. Un tel saut qualitatif ne saurait advenir sans que soit élaboré un "travail", portant sur les représentations, ancré dans un dispositif de fictionnalisation et reposant sur un support éditorial (un livre, une exposition, ou les deux à la fois). Cet article abordera la question de la résilience grâce en particulier à deux exemples, un roman de l'écrivain sud-africain Ivan Vladislavic (Double négatif, Zoé, 2013), associé au photographe David Goldblatt, et un ouvrage abondamment illustré de Leïla Sebbar (Mes Algéries en France, Bleu Autour, 2004).

If the photographic image is often linked to painful or traumatic events, whether personal or collective, and while the photographic act itself has often been associated with the idea of predation, it is necessary to wonder in what way and in what conditions this image, beyond its evidential or documentary value, may ensure the curative and pacifying dimension of resilience. Such a qualitative leap requires an actual "working process" based on representations, grounded in a fictionalizing apparatus and relying on an editorial aid (a book, an exhibition, or both). This essay will consider the question of resilience in two specific examples, a novel by South African writer Ivan Vladislavic (Double négatif, Zoé, 2013), in collaboration with photographer David Goldblatt, and an abundantly illustrated book by Leïla Sebbar (Mes Algéries en France, Bleu Autour, 2004).

\section{INDEX}

oeuvrecitee Mes Algéries en France, Double négatif

Keywords : photoliterature, resilience, trauma, Algeria, South Africa, France, portraits of countries, History, media, apparatus

Mots-clés : photolittérature, résilience, trauma, Algérie, Afrique du sud, France, portraits de pays, Histoire, médias, dispositif

\section{AUTEURS}

\section{JEAN-PIERRE MONTIER}

Jean-Pierre Montier est professeur à l'Université Rennes 2. Il a dirigé le Cellam (Centre d'études des littératures et langues anciennes et modernes) de 2003 à 2013, et ses recherches portent sur les relations entre littérature et photographie, via notamment le site créé avec Paul Edwards et Gyongyi Pal [www.phlit.org]. Il a publié nombre d'articles portant sur ces questions, concernant des auteurs tels que Baudelaire, Proust, Aragon, Éluard, Ricardou, et des photographes tels que Cartier-Bresson et Koudelka. Il a codirigé Revoir Cartier-Bresson (avec Anne Cartier-Bresson, 
Textuel, 2009) et Carrefour Stieglitz (avec Jay Bochner, PUR, 2012). Il a bénéficié, avec le Pr. S. Guerlac, d'une bourse du France Berkeley Fund en 2015 pour la promotion des recherches en photolittérature, et publié la même année Transactions photolittéraires (PUR). Il prépare une exposition sur ce sujet à la Fondation Jan Michalski, près de Lausanne. 